\title{
Perioperative characteristics and management of liver transplantation for isolated methylmalonic acidemia-the largest experience in China
}

\author{
Yi-Zhou Jiang ${ }^{1}$, Li-Ying Sun ${ }^{1,2}$, Zhi-Jun Zhu ${ }^{1}$, Lin Wei ${ }^{1}$, Wei Qu ${ }^{1}$, Zhi-Gui Zeng ${ }^{1}$, Ying Liu ${ }^{1}$, Yu-Le Tan ${ }^{1}$, \\ En-Hui He ${ }^{3}$, Rui-Fang Xu ${ }^{3}$, Liang Zhang ${ }^{4}$, Jun Wang ${ }^{1}$, Xiao-Jie Chen ${ }^{1}$ \\ ${ }^{1}$ Department of Liver Transplantation Center, ${ }^{2}$ Department of Intensive Care Unit, ${ }^{3}$ Department of Ultrasound, ${ }^{4}$ Department of Anesthesiology, \\ Beijing Friendship Hospital, Capital Medical University, Beijing 100050, China \\ Contributions: (I) Conception and design: ZJ Zhu, LY Sun; (II) Administrative support: LY Sun, ZJ Zhu; (III) Provision of study materials or patients: \\ All authors; (IV) Collection and assembly of data: All authors; (V) Data analysis and interpretation: YZ Jiang; (VI) Manuscript writing: All authors; (VII) \\ Final approval of manuscript: All authors. \\ Correspondence to: Li-Ying Sun. Department of Liver Transplantation Center and Intensive Care Unit, Beijing Friendship Hospital, Capital Medical \\ University, 95 Yong-An Road, Xi-Cheng District, Beijing 100050, China. Email: sunxlx@outlook.com.
}

Background: There are few detailed consensus and guidelines on perioperative clinical characteristics of liver transplantation (LT) in patients with methylmalonic acidemia (MMA). This retrospective study investigated details of the clinical course and individualized treatment plan of the center with largest experience in China.

Methods: A total of 7 MMA patients undergoing LT in Beijing Friendship Hospital from June 2013 to December 2017 were enrolled in the study, whose clinical data (clinical characteristics, laboratory findings, chronological changes in urine MMA levels, treatment, etc.) during perioperative period were analyzed retrospectively. All the patients received strict postoperative management.

Results: All the 7 cases were confirmed to have isolated MMA, among which, 3 cases received living donor liver transplantation (LDLT), 4 cases received deceased donor liver transplantation (DDLT). A wild fluctuate of metabolic condition was observed within the first few days after surgery and two weeks after LT, the mean base excess of blood value (BE-B) restored to normal whereas plasma bicarbonate $\left(\mathrm{HCO}_{3}{ }^{-}\right)$ was still below normal value even with intermittent sodium bicarbonate correction. It also showed marked reduction in propionylcarnitine $(\mathrm{C} 3)$ and $\mathrm{C} 3 / \mathrm{C} 2$ level and the mean urine MMA by gas chromatographymass spectrometry $(\mathrm{GC}-\mathrm{MS})$ was reduced by $81.7 \%(\mathrm{P}<0.01)$ but remained $>72 \times$ higher than upper limit of normal. The metabolism-correcting medications were administered as before. The renal function of one case with renal insufficiency before LT (serum creatinine rising) maintained stable by adjusting the immunosuppressive regimen during the observation period. All patients survive to date.

Conclusions: LT is an effective treatment to prevent metabolic crisis, but patients with MMA tend to be metabolically fragile even after surgery. During perioperative period, close monitoring should be given for acidosis episodes so as to implement sodium bicarbonate correction. Metabolism-correcting medications are still needed. Special immunosuppressive regimen is an effective way of maintaining renal function for those with kidney dysfunction.

Keywords: Methylmalonic acidemia (MMA); methylmalonic acid; liver transplantation (LT); perioperative management

Submitted Oct 12, 2018. Accepted for publication Jan 28, 2019.

doi: $10.21037 / \mathrm{hbsn} .2019 .03 .04$

View this article at: http://dx.doi.org/10.21037/hbsn.2019.03.04 


\section{Introduction}

Methylmalonic acidemia (MMA) (OMIM \#251000) is a rare autosomal recessive disorder of propionic acid metabolism induced by the deficiency of methylmalonylCoA mutase (MCM, the most common cause of MMA) or in the synthesis of adenosylcobalamin (cofactor), failing to transform L-methylmalonyl-CoA into succinylCoA (1). Inborn mutations in the gene MUT, located on chromosome $6 \mathrm{p} 21$ are responsible for the majority of cobalamin-unresponsive MCM deficiency (2). To date, 272 different mutations have been identified and most of them are missense/nonsense mutations [187] (3). This kind of errors of metabolism is presented with vast methylmalonic acid accumulated in plasma and urine with or without hyperhomocysteinemia (called isolated MMA).

Onset of the manifestations of isolated MMA ranges from the neonatal period to adulthood and the clinical symptoms are nonspecific. During neonatal period, the disease is usually characterized by feeding refusal, vomiting, hypotonia, hypothermia, hyperammonemia, severe ketoacidosis, lethargy and coma and even leads to death (4). Long-term signs and major complications include developmental delay, movement disorders (seizures, optic atrophy, et al.), chronic renal failure, cardiomyopathy $(5,6)$. Patients can experience recurrent and severe episodes of acute metabolic decompensation characterized by metabolic acidosis, hyperammonemia and progressive encephalopathy (7-9). The illness progresses rapidly if not promptly treated and the overall outcome remains poor.

Treatment should be started without delay with the combination of low protein diet avoiding an excess of isoleucine, methionine, threonine, valine, cholesterol, odd chain fatty acids and medications. Conventional long-term medical management of MMA includes oral levocarnitine supplementation, sodium bicarbonate, added with vitamin B12 in cobalamin-responsive MCM deficiency and sometimes human growth hormone as well. In addition, some known triggers of metabolic crises including infection and dehydration should also be avoided (10). However, long-term complications with multiple organ impairment especially kidney and nervous system are common in isolated MMA (11). Since most of the metabolic conversion of propionate occurs in the liver, replacing the liver could help regain enzyme activity and reduce circulating metabolites (12). After the first attempt in 1997 (13), liver transplantation (LT) has become a treatment option for patents with MMA, especially in treating severe MCM deficiency case. While this therapy is expected to prolong survival and improve the quality of life of patients, it does not bring about a complete cure (14). Problems such as metabolic stroke, progression of renal dysfunction and neurologic disability can still occur (15-17). Meanwhile, LT is a special procedure, which requires strict postoperative management including immunosuppressive therapy and close monitoring of a series of assay index. Consequently, it is vital to know what is unique about the MMA patient after LT and to take proper action when necessary.

Because of the lack of consensus and guidelines on perioperative clinical characteristics of LT in patients with MMA, any detailed description of the clinical course and individualized treatment plan experienced by any transplantation center may provide important information and unique insights, helping raise awareness and proposing timely intervention after such case undergoing a LT. In the present study, we describe the perioperative characteristics such as the underlying metabolic disorder of 7 patients with isolated MMA treated by LT in our center and share our management experience on some crucial issue.

\section{Methods}

Between June 2013 and December 2017, seven MMA pediatric patients underwent LT at liver transplantation center of Beijing Friendship Hospital, China. All patients were confirmed to have MMA by direct gene sequencing analysis and were on protein-restriction diet, a special formula that contains no methionine, threonine, valine and isoleucine, and were administered several metabolismcorrecting medications preoperatively. All MMA patients underwent a smooth operation and received strict postoperative management.

To explore the perioperative clinical characteristics of these seven patients, their clinical data such as clinical manifestation, serum creatinine level $\left(\mathrm{mmol} \cdot \mathrm{L}^{-1}\right)$, base excess of blood value (BE-B, mmol. $\mathrm{L}^{-1}$ ), plasma bicarbonate $\left(\mathrm{HCO}_{3}{ }^{-}, \mathrm{mmol} \cdot \mathrm{L}^{-1}\right)$, chronological changes in urine MMA levels (by C-MS) during perioperative period were analyzed retrospectively. For patient with pre-existing renal disfunction, we adjusted the immunosuppressive regimen for him. Our study was in accordance with the ethical standards and was approved by local ethics committee. Informed consent was obtained from the parents of all patients for being included in the study.

Statistical Product and Service Solutions (SPSS) version 20 statistics software was used for all statistical analyses. 
Table 1 Preoperative patient characteristics and operative procedures

\begin{tabular}{|c|c|c|c|c|c|c|c|c|c|c|}
\hline $\begin{array}{l}\text { Case } \\
\text { No. }\end{array}$ & Sex & $\begin{array}{l}\text { Age at } \\
\text { onset }\end{array}$ & $\begin{array}{l}\text { Current } \\
\text { age (yr) }\end{array}$ & $\begin{array}{l}\text { Major clinical } \\
\text { manifestation }\end{array}$ & Genetic analysis & Type & $\begin{array}{c}\text { Frequency of } \\
\text { acute metabolic } \\
\text { decompensation }\end{array}$ & $\begin{array}{c}\text { Serum Cr } \\
(\mathrm{mg} / \mathrm{dL})\end{array}$ & $\begin{array}{l}\text { Age at LT } \\
(y r)\end{array}$ & Procedure \\
\hline 2 & $\mathrm{~F}$ & $8 d$ & 3.2 & $\begin{array}{l}\text { Anorexia, somnolence, } \\
\text { recurrent vomiting }\end{array}$ & $\begin{array}{l}\text { Mut c. } 914 \text { c. } 1880 \\
\text { heterozygous mutations }\end{array}$ & Isolated $^{a}$ & NA & 0.5 & 2.5 & LDLT \\
\hline 3 & M & $6 \mathrm{~m}$ & 11.7 & $\begin{array}{l}\text { Anorexia, vomiting, } \\
\text { seizures and coma }\end{array}$ & $\begin{array}{l}\text { Mut c. } 1138 \text { G-A c. } 861 \\
\text { G-C heterozygous } \\
\text { mutations }\end{array}$ & Isolated $^{\mathrm{a}}$ & Once & $1.3^{b}$ & 11.0 & OLT \\
\hline 4 & M & $8 \mathrm{~m}$ & 7.5 & $\begin{array}{l}\text { High fever with nausea } \\
\text { and vomiting }\end{array}$ & $\begin{array}{l}\text { Mut c.729_730insTT } \\
\text { c. } 494 \text { A }>\text { G heterozygous } \\
\text { mutations }\end{array}$ & Isolated $^{\mathrm{a}}$ & NA & 0.8 & 7.0 & OLT \\
\hline 6 & M & $3 d$ & 6.2 & $\begin{array}{l}\text { Recurrent vomiting, } \\
\text { somnolence }\end{array}$ & $\begin{array}{l}\text { Mut c.729_730insTT } \\
\text { c.398_399del } \\
\text { heterozygous mutations }\end{array}$ & Isolated $^{a}$ & $1 / 1$ month & 0.8 & 5.9 & LDLT \\
\hline 7 & $\mathrm{~F}$ & $3 d$ & 4.1 & $\begin{array}{l}\text { Slow-response, } \\
\text { somnolence, low } \\
\text { temperature }\end{array}$ & $\begin{array}{l}\text { Mut c. } 2179 \mathrm{C}>\mathrm{T} \\
\text { c.729_730ins } T \mathrm{~T} \\
\text { heterozygous mutations }\end{array}$ & Isolated $^{a}$ & 1/1 year & 0.4 & 3.9 & LDLT \\
\hline
\end{tabular}

\footnotetext{
${ }^{a}$, patient does not manifest as combined homocystinuria; ${ }^{b}$, patient already had renal insufficiency before liver transplantation (serum
} creatine rising). NA, not available; Cr, creatinine; OLT, orthotopic liver transplantation; LDLT, living donor liver transplantation.

Statistical analysis data were represented as mean $(M) \pm$ standard deviation (SD). Statistical analyses were conducted using either the Student's $t$-test or the Mann-Whitney U test for comparative analysis between pre- and posts-LT. A $P$ value less than 0.05 was considered to be significant.

\section{Results}

\section{Preoperative patient characteristics \& operative procedures}

Preoperative patient characteristics and operative procedures are shown in Table 1 . All the 7 cases were confirmed to have isolated MMA, with various heterozygous mutations of the gene MUT. The average onset age of MMA of the 7 patients was $65.3 \pm 100.5$ days old (range, 3-240 days) and four of them (57.1\%) started showing symptoms in the neonatal period. The clinical manifestation was characterized mainly by anorexia, vomiting and altered level of consciousness, similar to those described previously. None of the seven patients had abnormal renal function before LT in view of serum creatinine, except case 3
(1.3 $\mathrm{mg} \cdot \mathrm{dL}^{-1}$, higher than normal). The frequency of acute metabolic decompensation varied from only once to one time a month and low-protein $\operatorname{diet}(<1.1 \mathrm{~g} / \mathrm{kg} / \mathrm{d})$ was maintained in all cases. Of these 7 patients, 3 received living donor liver transplantation (LDLT), the other 4 received deceased donor liver transplantation (DDLT). The average age at LT was $5.8 \pm 3.2$ years old. Immunosuppressive treatment consisted of tacrolimus and low-dose corticosteroids, and there was no major complication of the LT. To date, the overall patient and graft survival rate was $100 \%$ and $100 \%$, respectively.

\section{Metabolic consequences}

Metabolic acidosis is defined as the condition in which the $\mathrm{BE}$ is $-5 \mathrm{mmol} \cdot \mathrm{L}^{-1}$ or less, and metabolic stroke/crisis is an episode of faint caused by a sudden worsening of metabolic decompensation and the $\mathrm{BE}$ is generally $-10 \mathrm{mmol} \cdot \mathrm{L}^{-1}$ or less (18). For our seven patients, all had no metabolic strokes after LT anymore except case 1. 

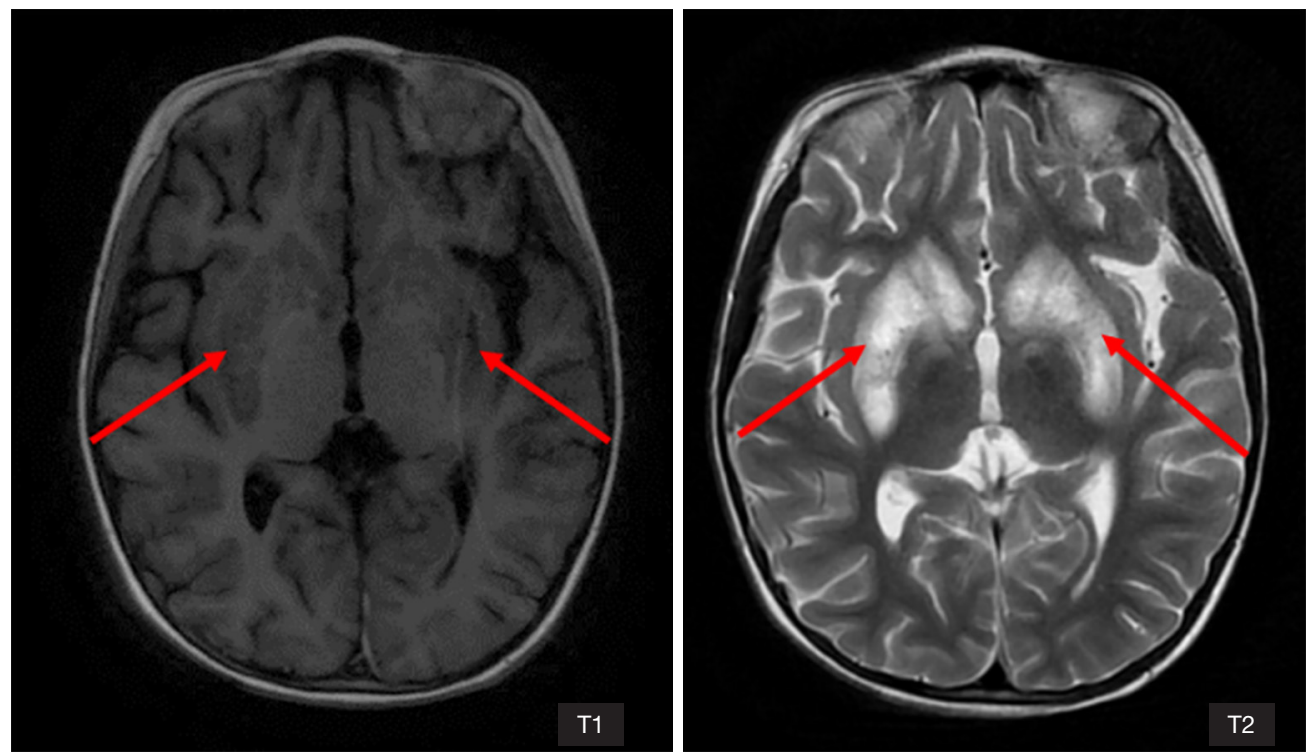

Figure 1 T1 (left) and T2-weighted (right) MRI scan of the brain 2 weeks after liver transplantation (1 week after onset of metabolic stroke) shows bilateral symmetrical abnormal low and high signal intensity of the basal ganglia (arrows), respectively. MRI, magnetic resonance imaging.

Case 1 became symptomatic on the fifth day after she was born, with vomiting and feeding difficulties. She was weak, even progressed to coma. The diagnosis of MMA was confirmed later and conventional metabolism-correcting medications were administered, accompanied by the restriction of natural protein intake. But the treatment did not bring about significant reduction in urine MMA, and the frequency of acute metabolic decompensation reached 1/1.5 month. During the 4 years preceding LT, she had been diagnosed with osteoporosis, and had femoral and humeral fractures twice, which have been described in association with the metabolic diet for MMA patients that is low in calcium and vitamin D levels (both of which are essential for bone health) $(19,20)$. With other complications such as developmental delay and damage of nervous system, she underwent LT at age 8 in Dec. 2014 in our center when urine MMA was as high as $1,160.59$. The operation went on smoothly and all the vital signs were stable during the process. She recovered consciousness and maintained circulation stabilization after being transferred to intensive care unit (ICU). Immunosuppression was instituted with tacrolimus (FK506) and methylprednisolone for antirejection of liver allograft. Albumin infusion and supportive anticoagulant and liver protection therapy were also used while serologic detections were monitored. On postoperative day 3 , when her condition became stable, the patient was transferred to ordinary ward to continue her conventional therapy. Five days later, she had a sudden altered consciousness with movement disorders of eyes. On the following day, an unexpected metabolic stroke appeared. Laboratory findings showed: $\mathrm{PH} 7.103, \mathrm{PCO}_{2} 50.3 \mathrm{mmHg}$, $\mathrm{HCO}_{3}{ }^{-} 15.3 \mathrm{mmol} / \mathrm{L}, \mathrm{BE}-13.8 \mathrm{mmol} / \mathrm{L}$. Metabolic acidosis combined with respiratory alkalosis was diagnosed, and $100 \mathrm{~mL}$ bicarbonate $(10 \%, 50 \mathrm{~mL} / \mathrm{h})$ was given by intravenous infusion as well as levocarnitine $(2 \mathrm{~g} / \mathrm{d})$ to enhance the elimination of organic acids. There was a downward trend of serum acidic_circulating metabolites levels with supplementation of bicarbonate. After acidosis was corrected, the patient had intermittent dystonic posturing disability of speaking and there was evidence of established symmetrical damage affecting the basal ganglia on magnetic resonance imaging (MRI, Figure 1). Levocarnitine and neurotrophic treatment were administered, and protein-restriction diet was fed through nasogastric feeding tube. On the day when she was discharged one month later, the patient had stable metabolic status and better clinical conditions (muscular tension, consciousness, limb activity, etc.), but was still unable to speak or swallow, needing nasogastric feeding tube to ensure feed tolerance.

In our later experience, blood gas analysis was monitored regularly for all cases even after LT, especially during ICU stay. Individual kinetics of BE-B \& bicarbonate over time and the amount of sodium bicarbonate correction during 

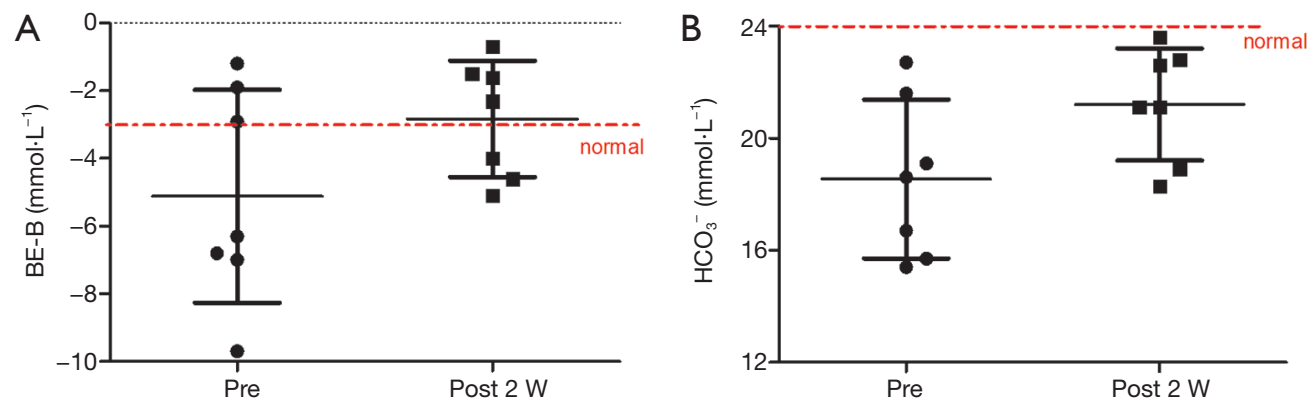

Figure 2 The value of BE-B (A) and plasma bicarbonate (B) pre- and post-LT (postoperative 2 weeks). BE-B, base excess of blood value; LT, liver transplantation.

the period of staying in ICU are shown in Figure S1. A wild fluctuate of metabolic condition was observed within the first few days after LT. When discharged from the ICU, all the patients were in stable condition.

Figure $2 A, B$ demonstrate the value of BE-B and plasma bicarbonate pre- and post-LT (postoperative 2 weeks). The average of BE-B and bicarbonate level before surgery were $-5.1 \pm 3.1$ and $18.5 \pm 2.8 \mathrm{mmol} / \mathrm{L}$, respectively. On postoperative week 2 , the mean $\mathrm{BE}-\mathrm{B}$ restored to normal with the value of $(-2.8 \pm 1.7) \mathrm{mmol} / \mathrm{L}$ whereas the level of bicarbonate was $21.20 \pm 2.00 \mathrm{mmol} / \mathrm{L}$, still lower than normal value even with intermittent sodium bicarbonate correction although an increase was observed compared with the preoperative period $(\mathrm{P}>0.05)$.

\section{Chronological changes in organic acids and metabolism- correcting medications}

As part of the routine examination for patients with MMA, urine organic acids and its precursor propionylcarnitine (C3) in blood are measured to monitor methylmalonic acid level and metabolic disorder. The mean $\mathrm{C} 3$ and $\mathrm{C} 3$ / C2 level before and after LT are shown in Figure $3 A, B$. Perioperative and longitudinal evaluations showed a marked reduction in $\mathrm{C} 3$ and $\mathrm{C} 3 / \mathrm{C} 2$ level, to $37.86 \pm 7.8 \mu \mathrm{mol} / \mathrm{L}$ and $0.60 \pm 0.32$ respectively and both are statistically significant $(\mathrm{P}=0.047$ and 0.01$)$, but still higher than the normal range (1.00-4.00 and 0.03-0.5). Figure 3C demonstrates preand post-LT ( 2 week) urine MMA level. Despite proteinrestriction and metabolism-correcting medications, the levels of urine MMA (by GC-MS) before LT maintained between 601.6 and $3,196.0$, with the mean value $1,420.0 \pm 1,052.0$ (normal range 0.2-3.6). Two weeks after LT, the MMA levels in urine were found to be decreased significantly, down to $18.3 \%$ of pre-LT levels $(260.4 \pm 191.9$,
$\mathrm{P}<0.01$ ), whereas it did not return to normal in any patient and still remained $>72 \times$ higher than upper limit of normal.

The main treatment, levocarnitine administered to enhance the elimination of organic acids, sodium bicarbonate tablets and special milk free of leucine, valine, threonine and methionine, vitamin B12 given to cobalamin responders remained unchanged after LT. More details see Table 2.

\section{Renal dysfunction}

All of the patients had a creatinine level within the normal range before LT except case 3. The application of proper dosage of immunosuppressants are required after transplantation to reduce the incidence of rejection. To slow down the progress to renal failure due to the use of immunosuppressive drugs soon after LT, we adjusted the immunosuppressive regimen for him.

Split-liver transplantation was performed at 11.7 years old in case 3 (see supplemental material), when he was $26 \mathrm{~kg}$. Treatment with methylprednisolone was initiated at first day after surgery; the dosage was tapered from 2.3 to $0.3 \mathrm{mg} / \mathrm{kg} /$ day $(15 \mathrm{mg} \mathrm{q} 6 \mathrm{~h}-12 \mathrm{mg} \mathrm{q} 6 \mathrm{~h}-10 \mathrm{mg} \mathrm{q} 6$ $\mathrm{h}-10 \mathrm{mg} \mathrm{q} 8 \mathrm{~h}-10 \mathrm{mg} \mathrm{q} 12 \mathrm{~h}-10 \mathrm{mg} \mathrm{qd}-8 \mathrm{mg} \mathrm{qd}$ ) in the first week and was maintained during his hospitalization. While tacrolimus (FK506) and mycophenolate mofetil (MMF) were not administered until $72 \mathrm{~h}$ postoperatively (normally $24-36 \mathrm{~h}$ ), with the doses of $0.075 \mathrm{mg} / \mathrm{kg} / \mathrm{day}$ $(1 \mathrm{mg} \mathrm{q} 12 \mathrm{~h})$ and $38.5 \mathrm{mg} / \mathrm{kg} /$ day $(500 \mathrm{mg} \mathrm{q} 12 \mathrm{~h}$ ), respectively. On postoperative day 7 , tacrolimus was added to $0.15 \mathrm{mg} / \mathrm{kg} /$ day $(2 \mathrm{mg} \mathrm{q} 12 \mathrm{~h}$ ) and remained so until day 12 when he was discharged.

The patient's serum creatinine levels and estimated glomerular filtration rate (eGFR) calculated according to simplified modification of diet in renal disease (MDRD) 

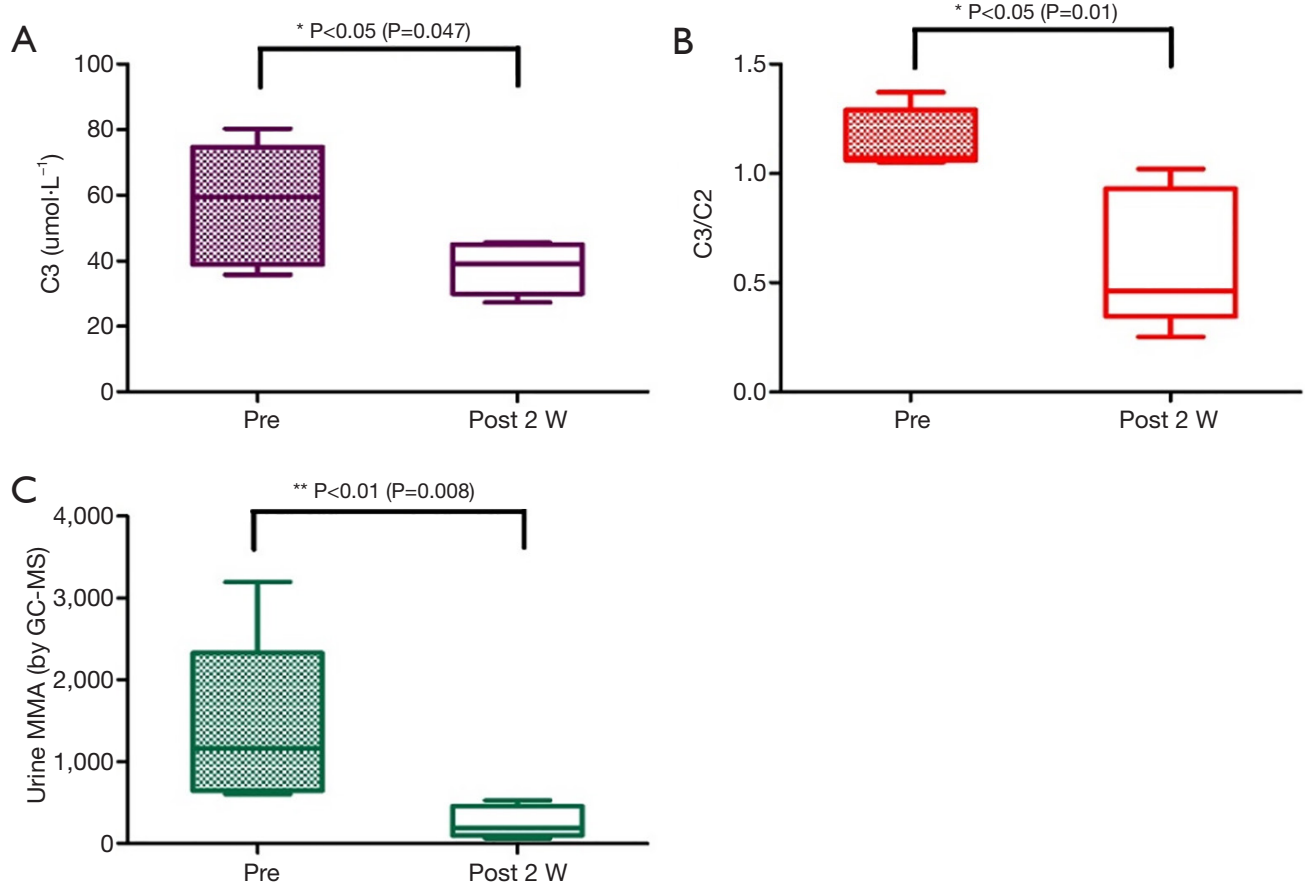

Figure 3 The mean C3 level (A), C3/C2 level (B) and urine MMA (C) pre- and post-LT (postoperative 2 weeks). Note that even after liver transplantation, all of them are still much above upper limit of normal. C3, propionylcarnitine.

Table 2 C3, C3/C2 and urine MMA level before and after LT

\begin{tabular}{|c|c|c|c|c|c|c|}
\hline \multirow{2}{*}{ Case No. } & \multicolumn{2}{|c|}{ C3 ( $\mu \mathrm{mol} / \mathrm{L})$} & \multicolumn{2}{|c|}{$\mathrm{C} 3 / \mathrm{C} 2$} & \multicolumn{2}{|c|}{ MMA (by GC-MS) } \\
\hline & Pre- & Post-2w & Pre- & Post-2w & Pre- & Post-2w \\
\hline 1 & NA & NA & NA & NA & 1160.59 & 392.04 \\
\hline 2 & 35.77 & 27.38 & 1.21 & 0.25 & 601.61 & 133.97 \\
\hline 3 & 69.08 & 32.67 & 1.05 & 0.44 & 1453.30 & 524.20 \\
\hline 5 & 80.35 & 45.45 & 1.07 & 1.02 & 1845.70 & - \\
\hline 6 & 41.93 & 39.22 & 1.07 & 0.46 & 687.94 & 61.68 \\
\hline 7 & 59.45 & 44.60 & 1.37 & 0.84 & 3196.27 & 190.10 \\
\hline
\end{tabular}

C3, propionylcarnitine; MMA, methylmalonic acidemia; LT, liver transplantation; GC-MS, gas chromatography-mass spectrometry; NA, not available.

equation pre-and post-LT are shown in Figure 4. Longitudinal renal function assessments showed stable $\mathrm{Cr}$ and eGFR, with a transient increase in the first few days after LT, followed by slight improvement up to pretransplant values. There were no episodes of rejection or surgical complications in perioperative period and the blood concentration of FK506 was 4.6, 9.2, $9.3 \mathrm{ng} /$ $\mathrm{mL}$ on postoperative day $5,10,14$, respectively. The liver function indices (ALT, AST, etc.) also recovered smoothly during observation period. At the time he was discharged, the patient had a creatinine of $1.2 \mathrm{mg} / \mathrm{dL}$ and an eGFR of $90.2 \mathrm{~mL} / \mathrm{min} / 1.73 \mathrm{~m}^{2}$.

\section{Discussion}

Isolated MMA is an inborn error of metabolism caused 

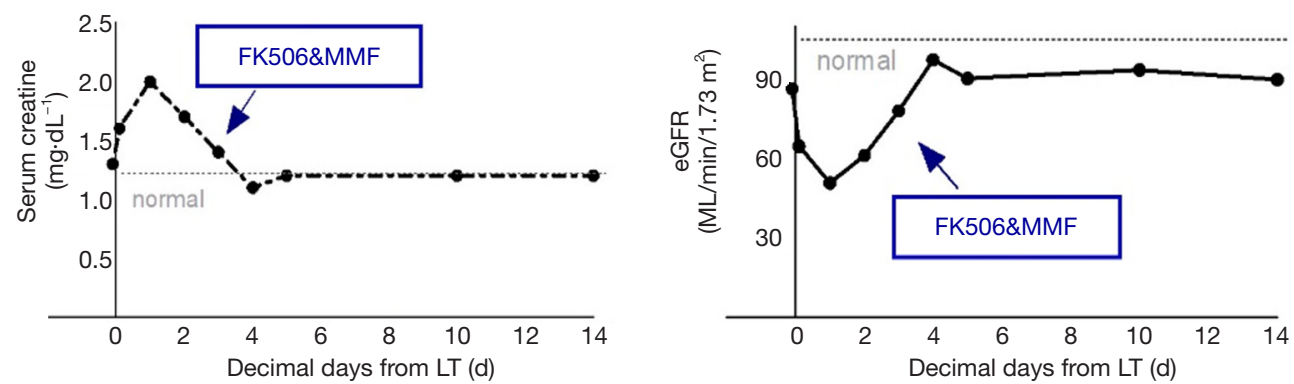

Figure 4 Serum creatine and eGFR in case 3 before and after LT. eGFR, estimated glomerular filtration rate; LT, liver transplantation.

either by a genetic defect in the MUT enzyme itself, or in one of the proteins involved in the synthesis of its active cofactor, adenosylcobalamin. LT has been more and more performed in patients with MMA to mitigate or eliminate metabolic crises. In our study, all patients had preoperative history of acute metabolic decompensation and/or metabolic stroke. After LT, case 1 still had an acute metabolic stroke due to the lack of experience in perioperative management for MMA patients. For case 2-7, blood gas analysis was monitored regularly even after LT. $\mathrm{BE}<-5.0 \mathrm{mmol} / \mathrm{L}$ usually suggests the worsening of clinical status and moderate dose of sodium bicarbonate shall be administered in case of metabolic strokes. But the specific time and amount of sodium bicarbonate correction for a certain patient need to take into account symptoms, other medications and previous conditions of metabolic disorder. After discharged from the ICU, metabolic correction was achieved mostly by using conventional biochemistry indexes instead of blood gas analysis for mitigating psychological stress, a trigger of acute decompensation (9). Under this circumstance, the mean bicarbonate value was still lower than normal value two weeks after LT. While no episode of acute metabolic stroke was observed postoperatively before discharged, proving the importance of maintaining keen vigilance of metabolic consequences and the effectiveness of our management. So, LT is a possible surgical procedure to reduce recurrent metabolic decompensation, in line with previous study (14). But patients with MMA tend to be metabolically fragile and a wild fluctuation of metabolic condition might still occur even after LT. Meanwhile, as our study showed, perioperative and longitudinal evaluations showed a marked reduction in urine MMA and its precursor C3. However, they did not return to normal in any patient and remained appreciably elevated above the upper limit of normal. This is consistent with previous study which showed although transplantation can substantially reduce the MMA concentration, metabolites remain more than 100 -fold higher compared to controls after successful transplantation (21). Metabolism-correcting medications remained mostly unchanged during perioperative period.

Liver provides the greatest mutase enzyme activity among all solid organs that transplantations are applied to, thus LT is a potential alternative for the management of severe MCM deficiency to help regain enzyme activity and reduce circulating metabolites (22). But since MMA is a systemic disease, the underlying enzymatic defect also exits in several other tissues besides liver. It is reported that, the widely distributed metabolic deficit may lead to a significant production of toxic compounds in many organs, particularly in central nervous system and kidney (15). The clinical manifestation is the result of the accumulation of toxic compounds in various organs including liver, kidney and brain. So, LT is just a partially curative procedure with the abnormal phenotype still maintained by extrahepatic synthesis of the toxic metabolites (23). In our cases, we didn't detect the MMA levels in cerebrospinal fluid before and after LT due to constrained condition. But according to other studies, the value of MMA in cerebrospinal fluid almost remained unchanged after LT (13). Meanwhile, it is revealed in other studies that patients with severe MMA are at high risk at having damage in basal ganglia, although the concrete mechanism is still unclear (21). One hypothesis is that the basal ganglia requires high energy in children (24) and more likely to be injured when brain energy metabolism impaired. Some mechanisms have been proposed that methylmalonate in MMA patients that inhibits aerobic oxidation may harmfully affect energy production of brain. Potential persistent intrathecal methylmalonate synthesis is considered a permanent risk factor for metabolic stroke $(15,25)$. In rats' model $(25)$, methylmalonate and other accumulating metabolites are found to have underlying direct neurotoxic effects with the proof that they can induce 
rotational behavior and convulsions through glutaminergic mechanisms (26). Based on those studies, we can infer that patients can present with metabolic stroke and have severe motor disabilities even after LT especially when acute and chronic basal ganglia damage are involved. It is reasonable to assume that metabolites existing in brain are difficult to get out of cerebrospinal fluid, in consistent with what was suggested in previous study that suggested autonomous production of MMA in the brain could not be transported across the blood brain barrier (27), thus the nerve damage caused by MMA may be irreversible since the cerebral MMA level after LT possibly remains still high and toxic to the brain (28). However, it is a pity that in our study, case 1 did not have the neuroimaging before LT, thus we cannot conduct a comparison with the one performed when she had a metabolic stroke after LT. Nevertheless, our study provides further evidence that urine MMA does not reflect actual MMA concentration in CSF and marked reduction in urine MMA and plasma C3 level cannot totally prevent neurological complications. The relationships between them and the approach of reducing/removing cerebral MMA and reversing neurological damage still needs to be further investigated. And when nervous system is involved, the neuroimaging is necessary before and after LT. With comparison, the outcomes of LT in neurologic disability can be much clearer. The correlation of indication in neuroimaging and severity of diseases may contribute to accurate timing of LT for individual cases.

The regimen mainly follows the principle of strict dietary restriction of the precursor amino acids and administration of levocarnitine that removes toxic metabolites. During perioperative period of LT, the dosage remained same in our study. In addition, since in vitro experiments, animal model studies and tissue analyses from human patients suggested that mitochondrial energy metabolism impairment exists in MMA patients through a combination of the inhibition of specific enzymes and transporters (29), some specific mitochondrially-targeted antibiotics such as the erythromycins, the tetracyclines, the glycylcyclines and chloramphenicol (30) should be avoided. The detailed data will be observed in a long run.

In addition, individuals with isolated MMA are also at risk of long-term complications of renal disease $(25,27)$. Since kidneys can only be partially protected through LT, renal function still needs to be monitored even after LT. Considering the renal failure is sometimes even accelerated by taking immunosuppressive drugs after LT (9), we postponed the initial time of using FK506 and MMF to
$72 \mathrm{~h}$ postoperatively for case 3 who had already suffered from renal dysfunction before LT. The result shows, renal functions within two weeks after LT were stable except an acute renal impairment occurred the first day postoperatively which we considered having association with hypoperfusion and his unable to urinate soon after surgery.

Overdose of immunosuppression can cause deterioration of renal function and other fatal side effects after LT while inadequate immunosuppression may lead to a consequent graft rejection (31). Therefore, monitoring the patients' immune function and personalizing clinical dosage of immunosuppression for those have low eGFR before LT is particularly important. In our experience, FK506 level shall be maintained in the therapeutic range between $8-10 \mathrm{ng} / \mathrm{mL}$ in the initial postoperative period (administered between 24-36 h postoperatively). But for patients with pre-existing renal dysfunction, the criterion should be broadened properly. It came out to be a success of our experimental immunosuppressive regimen as there were no episodes of rejection or surgical complications in perioperative period nor exacerbation of renal function as well in case 3.

Chronic kidney disease due to progressive tubulointerstitial injury manifests frequently in MMA patients and may eventually results in the need for dialysis or kidney transplantation (32). In a mut0 MMA case undergoing combined liver/kidney transplantation reported in other studies (27), peri-operative hemodialysis did not stop the acute deterioration of pre-existing chronic bilateral optic neuropathy although a stable post-operative reduction in plasma MMA concentration was observed. So far, neither dialysis nor kidney transplantation is the radical solution for MMA patients with renal failure. Therefore, the best way is not to let the disease deteriorate to renal failure. However, renal dysfunction is difficult to diagnose and monitor early in life since most patients with MMA maintain low muscle mass and are pediatric patients, serum creatinine may be an imprecise indicator of renal function. eGFR using simplified MDRD equation does not adjust as per body mass too and may lead to high data error when the values approach $90 \mathrm{~mL} / \mathrm{min} / 1.73 \mathrm{~m}^{2}(33,34)$. So, a better alternative equation is needed in order to give an accurate estimation of GFR for MMA pediatric patients thus avoid the need for combined kidney transplants (recommended when eGFR $<20 \mathrm{~mL} / \mathrm{min} / 1.73 \mathrm{~m}^{2}$ ). Whether the renal dysfunction does not reverse after LT still requires long-term observation.

Our study has a number of limitations. This is a 
retrospective, single-center study and the sample size is comparatively small. Secondly, a few data are unavailable since we were still on the road of exploring the perioperative clinical characteristics and management experience of LT in patients with isolated MMA. All patients are currently doing well in response to the LT. In the future, developmental delay, protein tolerance, medications and neurodevelopmental status need to be observed in a long run to explore the efficacy of LT in patients with MMA, and we are doing so.

\section{Conclusions}

MMA has its particularity in perioperative characteristics and managements after LT. A better understanding is critical in reaching consensus and guidelines on effective perioperative management modalities for MMA so as to protect patients from severe perioperative decompensation and long-term sequelae. This report provides some detailed descriptions of the clinical course and individualized treatment experience of our center, proving that LT does not bring about a complete cure for isolated MMA patients. During perioperative period, close monitoring should be given for acidosis decompensation and timely intervention is effective in avoiding metabolic stroke. Metabolismcorrecting medications are still needed even after surgery. Our experience also provides evidence that a special immunosuppressive regimen is feasible to maintain renal function for those with pre-existing kidney damage. The correlation of indication in neuroimaging and metabolic decompensation still requires further investigation.

\section{Acknowledgments}

Funding: This work was supported by The Capital Health Research and Development of Special (No.2016-1-2021); and Beijing Municipal Administration of Hospitals Ascent Plan (Code: DFL20150101).

\section{Footnote}

Conflicts of Interest: The authors have no conflicts of interest to declare.

Ethical Statement: The study protocol was approved by the ethics committee review board of Beijing Friendship Hospital, Capital Medical University. Informed consent was obtained from all patients for surgical treatment. Data collection and analysis were performed according to the ethical standards of the Helsinki Declaration (Approval ID: BJFH-EC/2013-042).

\section{References}

1. Willard HF, Rosenberg LE. Inherited methylmalonyl CoA mutase apoenzyme deficiency in human fibroblasts: evidence for allelic heterogeneity, genetic compounds, and codominant expression. J Clin Invest 1980;65:690-8.

2. Ledley FD, Lumetta MR, Zoghbi HY, et al. Mapping of human methylmalonyl CoA mutase (MUT) locus on chromosome 6. Am J Hum Genet 1988;42:839-46.

3. Keyfi F, Talebi S, Varasteh AR. Methylmalonic Acidemia Diagnosis by Laboratory Methods. Rep Biochem Mol Biol 2016;5:1-14.

4. de Baulny HO, Benoist JF, Rigal O, et al. Methylmalonic and propionic acidaemias: management and outcome. J Inherit Metab Dis 2005;28:415-23.

5. Prada CE, Al Jasmi F, Kirk EP, et al. Cardiac disease in methylmalonic acidemia. J Pediatr 2011;159:862-4.

6. Traber G, Baumgartner MR, Schwarz U, et al. Subacute bilateral visual loss in methylmalonic acidemia. J

Neuroophthalmol 2011;31:344-6.

7. Spada M, Calvo PL, Brunati A, et al. Early Liver Transplantation for Neonatal-Onset Methylmalonic Acidemia. Pediatrics 2015;136:e252-6.

8. Baumgarter ER, Viardot C. Long-term follow-up of 77 patients with isolated methylmalonic acidaemia. J Inherit Metab Dis 1995;18:138-42.

9. Baumgartner MR, Hörster F, Dionisi-Vici C, et al. Proposed guidelines for the diagnosis and management of methylmalonic and propionic acidemia. Orphanet J Rare Dis 2014;9:130.

10. Wendel U, Ogier de Baulny H. Branched-Chain Organic Acidurias/Acidemias. In: Inborn Metabolic Diseases 4 ed Heidelberg Germany. Springer, 2006:246-62.

11. van der Meer SB, Poggi F, Spada M, et al. Clinical outcome of long-term management of patients with vitamin B12-unresponsive methylmalonic acidemia. J Pediatr 1994;125:903-8.

12. Leonard JV. The management and outcome of propionic and methylmalonic acidaemia. J Inherit Metab Dis 1995;18:430-4.

13. Goyens P, Brasseur D, Otte JB, et al. Liver transplantation for methylmalonyl CoA mutase deficiency. J Inherit Metab Dis $1997 ; 20: 38$.

14. van't Hoff W, McKiernan PJ, Surtees RA, et al. Liver 
transplantation for methylmalonic acidaemia. Eur J Pediatr 1999;158 Suppl 2:S70-4.

15. Nyhan WL, Gargus JJ, Boyle K, et al. Progressive neurologic disability in methylmalonic acidemia despite transplantation of the liver. Eur J Pediatr 2002;161:377-9.

16. Burdelski M, Ullrich K. Liver transplantation in metabolic disorders: summary of the general discussion. Eur J Pediatr 1999;158:S95-6.

17. Kamei K, Ito S, Shigeta T, et al. Preoperative dialysis for liver transplantation in methylmalonic acidemia. Ther Apher Dial 2011;15:488-92.

18. Morioka D, Kasahara M, Horikawa R, et al. Efficacy of Living Donor Liver Transplantation for Patients With Methylmalonic Acidemia. Am J Transplant 2007;7:2782-7.

19. North KN, Korson MS, Gopal YR, et al. Neonatalonset propionic acidemia: neurologic and developmental profiles, and implications for management. J Pediatr 1995;126:916-22.

20. Pena L, Franks J, Chapman KA, et al. Natural history of propionic acidemia. Mol Genet Metab 2012;105:5-9.

21. Chakrapani A, Sivakumar P, McKiernan PJ, et al. Metabolic stroke in methylmalonic acidemia five years after liver transplantation. J Pediatr 2002;140:261-3.

22. Andrews E, Jansen R, Crane AM, et al. Expression of recombinant human methylmalonyl-CoA mutase: in primary mut fibroblasts and Saccharomyces cerevisiae. Biochem Med Metab Biol 1993;50:135-44.

23. Fagiuoli S, Daina E, D'Antiga L, et al. Monogenic diseases that can be cured by liver transplantation. J Hepatol 2013;59:595-612.

24. Smith CB, Sokoloff L. The energy metabolism of the brain. In: Davidson AN, Thompson RH, editors. The molecular basis of neuropathology. London: Edward Arnold Limited; 1981.

25. Kaplan P, Ficicioglu C, Mazur AT, et al. Liver transplantation is not curative for methylmalonic

Cite this article as: Jiang YZ, Sun LY, Zhu ZJ, Wei L, Qu W, Zeng ZG, Liu Y, Tan YL, He EH, Xu RF, Zhang L, Wang J, Chen XJ. Perioperative characteristics and management of liver transplantation for isolated methylmalonic acidemiathe largest experience in China. HepatoBiliary Surg Nutr 2019;8(5):470-479. doi: 10.21037/hbsn.2019.03.04 acidopathy caused by methylmalonyl-CoA mutase deficiency. Mol Genet Metab 2006;88:322-6.

26. de Mello CF, Begnini J, Jimenez-Bernal RE, et al. Intrastriatal methylmalonic acid administration induces rotational behavior and convulsions through glutamatergic mechanisms. Brain Res 1996;721:120-5.

27. Vernon HJ, Sperati CJ, King JD, et al. A detailed analysis of methylmalonic acid kinetics during hemodialysis and after combined liver/kidney transplantation in a patient with mut 0 methylmalonic acidemia. J Inherit Metab Dis 2014;37:899-907.

28. Chen PW, Hwu WL, Ho MC, et al. Stabilization of blood methylmalonic acid level in methylmalonic acidemia after liver transplantation. Pediatr Transplant 2010;14:337-41.

29. Melo DR, Kowaltowski AJ, Wajner M, et al. Mitochondrial energy metabolism in neurodegeneration associated with methylmalonic acidemia. J Bioenerg Biomembr 2011;43:39-46.

30. Lamb R, Ozsvari B, Lisanti CL, et al. Antibiotics that target mitochondria effectively eradicate cancer stem cells, across multiple tumor types: treating cancer like an infectious disease. Oncotarget 2015;6:4569-84.

31. Israeli M, Klein T, Sredni B, et al. ImmuKnow: a new parameter in immune monitoring of pediatric liver transplantation recipients. Liver Transpl 2008;14:893-8.

32. Kruszka PS, Manoli I, Sloan JL, et al. Renal growth in isolated methylmalonic acidemia. Genet Med 2013;15:990-6.

33. Rule AD, Larson TS, Bergstralh EJ, et al. Using serum creatinine to estimate glomerular filtration rate: accuracy in good health and in chronic kidney disease. Ann Intern Med 2004;141:929-37.

34. Levey AS, Coresh J, Greene T, et al. Using standardized serum creatinine values in the modification of diet in renal disease study equation for estimating glomerular filtration rate. Ann Intern Med 2006;145:247-54. 


\section{Supplementary}

Case 2

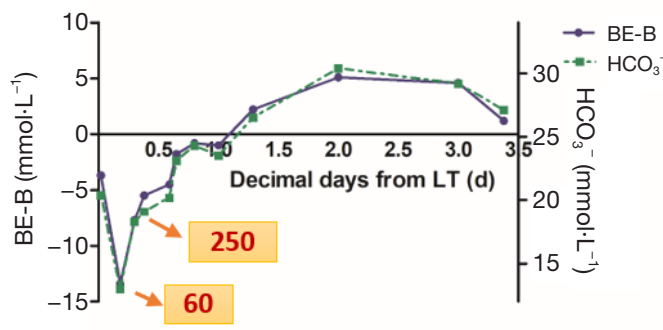

Case 4

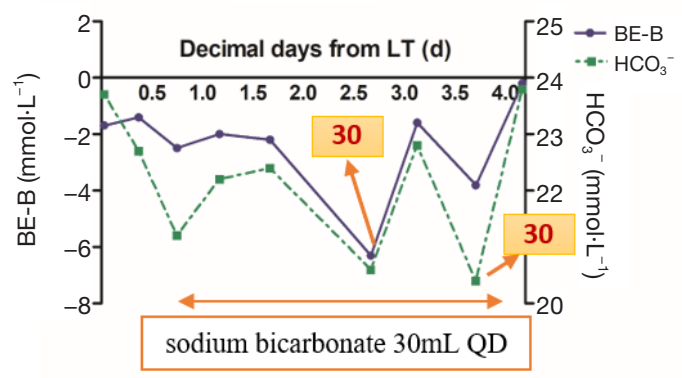

Case 6

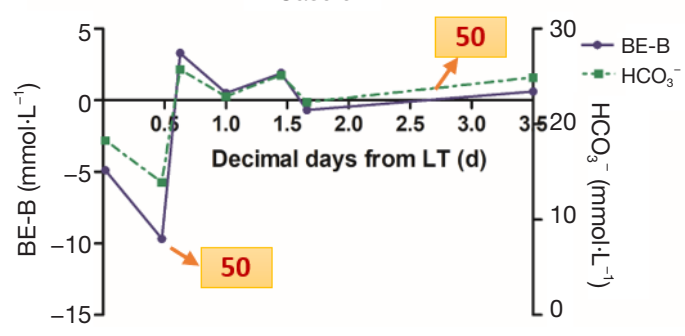

Case 3

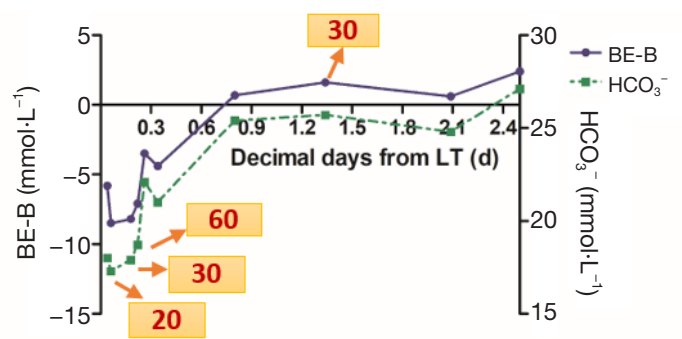

Case 5

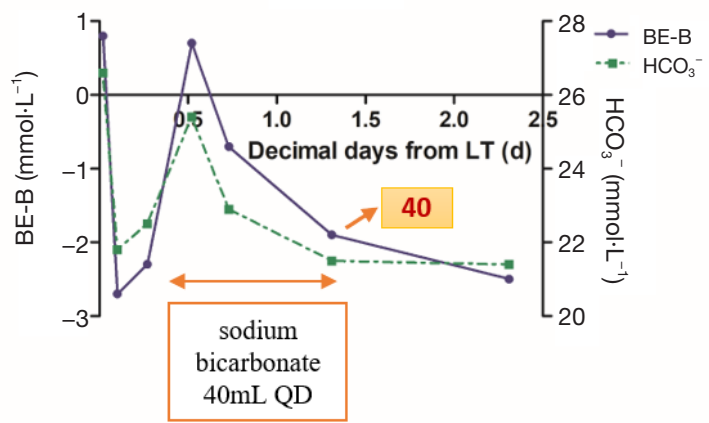

Case 7

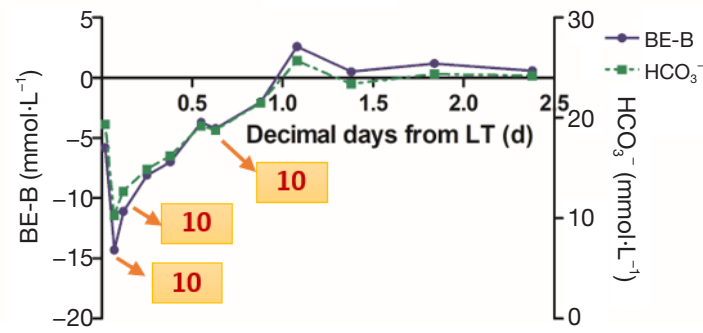

Figure S1 Individual kinetics of BE-B \& bicarbonate over time and the amount of sodium bicarbonate correction (mL, 5\%, iv.gtt). BE-B, base excess of blood value. 